\title{
Solvability for a nonlinear third-order three-point boundary value problem
}

\author{
Habib Djourdem $^{\mathrm{a}^{*}}$ and Slimane Benaicha ${ }^{\mathrm{a}}$ \\ ${ }^{\mathrm{a}}$ Laboratory of Fundamental and Applied Mathematics of Oran (LMFAO), University of Oran1, Ahmed Benbella, Algeria \\ *Corresponding author E-mail: djourdem.habib7@gmail.com
}

\section{Article Info}

Keywords: Positive solutions, nonlocal integral boundary value problem, Concavity, Leray-Schauder fixed point theorem.

2010 AMS: $34 B 15,34 B 18$

Received: 1 March 2018

Accepted: 12 April 2018

Available online: 26 June 2018

\begin{abstract}
In this article, the existence of positive solutions for a nonlinear third-order three-point boundary value problem with integral condition is investigated. By using Leray-Schauder fixed point theorem, sufficient conditions for the existence of at least one positive solution are obtained. Illustrative examples are also presented to show the applicability of our results.
\end{abstract}

\section{Introduction}

This paper is devoted to the existence of positive solutions for the following third-order nonlocal integral boundary value problem (BVP):

$$
\begin{aligned}
& u^{\prime \prime \prime}(t)+a(t) f(t, u(t))=0, \quad 0<t<T, \\
& u(0)=u^{\prime \prime}(0)=0, u(T)=\alpha \int_{0}^{\eta} u(s) d s,
\end{aligned}
$$

where $0<\eta<T, 0<\alpha<\frac{2 T}{\eta^{2}}$ and

$\left(H_{1}\right) f([0, T] \times[0,+\infty),[0,+\infty))$

$\left(H_{2}\right) a \in C([0, T],[0,+\infty))$ and there exists $t_{0} \in[\eta, T]$ such that $a\left(t_{0}\right)>0$.

Set

$$
f_{0}=\lim _{u \rightarrow 0^{+}} \frac{f(t, u)}{u}, \quad f_{\infty}=\lim _{u \rightarrow \infty} \frac{f(t, u)}{u},
$$

then $f_{0}=0$ and $f_{\infty}=\infty$ correspond to the superlinear case, $f_{0}=\infty$ and $f_{\infty}=0$ correspond to the sublinear case.

Third-order boundary-value problems for differential equations arise in variety of different areas of applied mathematics and physics. They have been many scholars' research object. For example, heat conduction, chemical engineering, underground water flow, thermoelasticity, and plasma physics can produce boundary-value problems with integral boundary conditions; see [3, 9, 11]. They include two, three, multipoint, and nonlocal boundary-value problems as special cases. By using the Krasnoselskii's fixed point theorem, Liu and Ma [19] studied the problem

$$
u^{\prime \prime \prime}(t)+f(u(t))=0, \quad 0<t<1,
$$


subject to integral boundary condition of the form

$$
u^{\prime}(0)=0, u^{\prime}(1)=0, u(0)=\int_{0}^{1} k(s) u(s) d s .
$$

Benaicha and Haddouchi [17] considered the fourth-order two-point boundary value problem

$$
\begin{aligned}
& u^{\prime \prime \prime \prime}(t)+f(u(t))=0, \quad t \in(0,1) \\
& u^{\prime}(0)=u^{\prime}(1)=u^{\prime \prime}(0)=0, u(0)=\int_{0}^{1} a(s) u(s) d s .
\end{aligned}
$$

We quote also the reasearchs of $[2,4,5,6,7,8,12,13,14,15,16,18,20]$ which concern the differential equations under various boundary conditions and by different approaches.

Motivated by the works mentioned above, we obtain the existence results for the problem (1.1)-(1.2) by using the Leray-Shauder fixed point theorem if $f_{0}=0$ ( condition $f_{\infty}=\infty$ being unnecessary ), as well as, for $f_{\infty}=0$ ( condition $f_{0}=\infty$ being unnecessary ). In this way we remove the half of the assumptions to prove the existence of a solution when using Krasnoselskii's fixed point theorem.(See [10, 17, 19]). Moreover, we establish our results for $t$ in $[0, T]$.

Our main tool is the following Leray-Schauder fixed point theorem.

Theorem 1.1. [1] Let $\Omega$ be the convex subset of Banach space $E, 0 \in \Omega, \Phi: \Omega \rightarrow \Omega$ be completely continuous operator. Then, either (i) $\Phi$ has at least one fixed point in $\Omega$;

or

(ii) the set $\{x \in \Omega \mid u=\lambda \Phi u, 0<\lambda<1\}$ is unbounded.

\section{Background}

To prove the main existence results we will employ several straightforward lemmas.

Lemma 2.1. Let $2 T \neq \alpha \eta^{2}$. Then for $y \in C([0, T],[0, \infty))$, the problem

$$
u^{\prime \prime \prime}(t)+y(t)=0,
$$

$$
u(0)=u^{\prime \prime}(0)=0, u(T)=\alpha \int_{0}^{\eta} u(s) d s, \quad \eta \in(0, T), \quad \alpha>0,
$$

has a unique solution given by

$$
\begin{aligned}
u(t)= & \frac{t}{2 T-\alpha \eta^{2}} \int_{0}^{T}(T-s)^{2} y(s) d s-\frac{\alpha t}{3\left(2 T-\alpha \eta^{2}\right)} \int_{0}^{\eta}(\eta-s)^{3} y(s) d s \\
& -\frac{1}{2} \int_{0}^{t}(t-s)^{2} y(s) d s .
\end{aligned}
$$

Proof. From equation (2.1) we have $u^{\prime \prime \prime}(t)=-y(t)$. Then, integrating from 0 to $t$ we obtain

$$
u^{\prime \prime}(t)=-\int_{0}^{t} y(s) d s .
$$

For $t \in[0, T]$ we have, by integrating in $t$ and using integration by parts,

$$
\begin{aligned}
u^{\prime}(t) & =u^{\prime}(0)-\int_{0}^{t}\left(\int_{0}^{x} y(s) d s\right) d x \\
& =u^{\prime}(0)-\int_{0}^{t}(t-s) y(s) d s \\
u(t) & =u^{\prime}(0) t-\int_{0}^{t}\left(\int_{0}^{x}(x-s) y(s) d s\right) d x \\
& =u^{\prime}(0) t-\frac{1}{2} \int_{0}^{t}(t-s)^{2} y(s) d s .
\end{aligned}
$$

Thus, for $t=T$ we find

$$
u(T)=u^{\prime}(0) T-\frac{1}{2} \int_{0}^{T}(T-s)^{2} y(s) d s .
$$

Integrating again from 0 to $\eta$ the expression (2.3), where $\eta \in(0, T)$, we obtain

$$
\begin{gathered}
\int_{0}^{\eta} u(s) d s=\frac{1}{2} u^{\prime}(0) \eta^{2}-\frac{1}{2} \int_{0}^{\eta}\left(\int_{0}^{x}(x-s)^{2} y(s) d s\right) d x \\
=\frac{1}{2} u^{\prime}(0) \eta^{2}-\frac{1}{6} \int_{0}^{\eta}(\eta-s)^{3} y(s) d s .
\end{gathered}
$$


From (2.2) and (2.4) we have

$$
\int_{0}^{\eta} u(s) d s=\frac{1}{\alpha} u(T)=u^{\prime}(0) \frac{T}{\alpha}-\frac{1}{2 \alpha} \int_{0}^{T}(T-s)^{2} y(s) d s .
$$

Then, using (2.5) we see that

$$
u^{\prime}(0) \frac{T}{\alpha}-\frac{1}{2 \alpha} \int_{0}^{T}(T-s)^{2} y(s) d s=\frac{1}{2} u^{\prime}(0) \eta^{2}-\frac{1}{6} \int_{0}^{\eta}(\eta-s)^{3} y(s) d s .
$$

Thus,

$$
u^{\prime}(0)\left(\frac{2 T-\alpha \eta^{2}}{2 \alpha}\right)=\frac{1}{2 \alpha} \int_{0}^{T}(T-s)^{2} y(s) d s-\frac{1}{6} \int_{0}^{\eta}(\eta-s)^{3} y(s) d s
$$

or

$$
u^{\prime}(0)=\frac{1}{\left(2 T-\alpha \eta^{2}\right)} \int_{0}^{t}(T-s)^{2} y(s) d s-\frac{\alpha}{3\left(2 T-\alpha \eta^{2}\right)} \int_{0}^{\eta}(\eta-s)^{3} y(s) d s .
$$

Therefore, the BVP (2.1)-(2.2) has a unique solution

$$
\begin{aligned}
u(t)= & \frac{t}{2 T-\alpha \eta^{2}} \int_{0}^{T}(T-s)^{2} y(s) d s-\frac{\alpha t}{3\left(2 T-\alpha \eta^{2}\right)} \int_{0}^{\eta}(\eta-s)^{3} y(s) d s \\
& -\frac{1}{2} \int_{0}^{t}(t-s)^{2} y(s) d s .
\end{aligned}
$$

The existence of positive solutions of the problem (2.1)-(2.2) is given in the next result.

Lemma 2.2. . Let $0<\alpha<\frac{2 T}{\eta^{2}}$. If $y \in C([0, T],[0,+\infty))$, then the unique solution of the problem (2.1)-(2.2) satisfies $u(t) \geq 0$ for $t \in[0, T]$.

Proof. From $u^{\prime \prime \prime}(t)=-y(t), t \in[0, T]$, we get that $u^{\prime \prime}(t)$ is decreasing on $[0, T]$. Then, the condition $u^{\prime \prime}(0)=0$ ensures that have $u^{\prime \prime}(t) \leq 0$, $t \in[0, T]$, which implies $u(t)$ is concave. Observe also that if $u(T) \geq 0$, the concavity of $u$ and the fact that $u(0)=0$ imply that $u(t) \geq 0$ for $t \in[0, T]$.

Since the graph of $u$ is concave down $(0, T)$, we get

$$
\int_{0}^{\eta} u(s) d s \geq \frac{1}{2} \eta u(\eta)
$$

where $\frac{1}{2} \eta u(\eta)$ is the area of triangle under the curve $u(t)$ from $t=0$ to $t=\eta$ for $\eta \in(0, T)$.

If we assume that $u(T)<0$, then from 2.2 we have

$$
\int_{0}^{\eta} u(s) d s<0
$$

By concavity of $u$ and $\int_{0}^{\eta} u(s) d s<0$, it implies that $u(\eta)<0$.

Hence

$$
u(T)=\alpha \int_{0}^{\eta} u(s) d s \geq \frac{2 T}{\eta^{2}} \times \frac{1}{2} \eta u(\eta)=\frac{T}{\eta} u(\eta),
$$

which contradicts the concavity of $u$.

Lemma 2.3. Let $\alpha>\frac{2 T}{\eta^{2}}$. If $y \in C([0, T],[0,+\infty))$, then the problem (2.1)-(2.2) has no positive solution.

Proof. Suppose that the problem (2.1)-(2.2) has a positive solution $u$.

If $u(T)>0$, then $\int_{0}^{\eta} u(s) d s>0$. It implies that $u(\eta)>0$ and

$$
\frac{u(T)}{T}=\frac{\alpha}{T} \int_{0}^{\eta} u(s) d s>\frac{2}{\eta^{2}}\left(\frac{1}{2} \eta u(\eta)\right)=\frac{u(\eta)}{\eta}
$$

This contradicts the concavity of $u$.

If $u(T)=0$, then $\int_{0}^{\eta} u(s) d s=0$, this is $u(t) \equiv 0$ for all $t \in[0, \eta]$. If there exists $t_{0} \in(\eta, T)$ such that $u\left(t_{0}\right)>0$, then $u(0)=u(\eta)<u\left(t_{0}\right)$, which contradicts the concavity of $u$. Therefore, no positive solutions exist.

Lemma 2.4. . Let $0<\alpha<\frac{2 T}{\eta^{2}}$. If $y \in C([0, T],[0,+\infty))$, then the unique solution of the problem (2.1)-(2.2) satisfies

$$
\min _{t \in[\eta, T]} u(t) \geq \gamma\|u\|,\|u\|=\max _{t \in[0, T]}|u(t)|,
$$

where

$$
\gamma:=\min \left\{\frac{\eta}{T}, \frac{\alpha \eta^{2}}{2 T}, \frac{\alpha \eta(T-\eta)}{2 T-\alpha \eta^{2}}\right\}
$$


Proof. Set $u(\tau)=\|u\|$. We consider three cases.

Case 1. If $\eta \leq \tau \leq T$ and $\min _{t \in[\eta, T]} u(t)=u(\eta)$, then the concavity of $u$ implies that

$$
\frac{u(\eta)}{\eta} \geq \frac{u(\tau)}{\tau} \geq \frac{u(\tau)}{T}
$$

Thus,

$$
\min _{t \in[\eta, T]} u(t) \geq \frac{\eta}{T}\|u\|
$$

Case 2. If $\eta \leq \tau \leq T$ and $\min _{t \in[\eta, T]} u(t)=u(T)$, then (2.2)-(2.6) and the concavity of $u$ implies

$$
\left.u(T)=\alpha \int_{0}^{\eta} u(s) d s \geq \alpha \frac{\eta^{2}}{2}\left[\frac{u(\eta)}{\eta}\right] \geq \alpha \frac{\eta^{2}}{2}\left[\frac{u(\tau)}{\tau}\right]\right) \geq \frac{\alpha \eta^{2}}{2 T} u(\tau)
$$

Therefore,

$$
\min _{t \in[\eta, T]} u(t) \geq \frac{\alpha \eta^{2}}{2 T}\|u\| .
$$

Case 3. If $\tau \leq \eta \leq T$, then $\min _{t \in[\eta, T]} u(t)=u(T)$. Using the concavity of $u$ and (2.2)-(2.6), we have

$$
\begin{gathered}
\frac{u(\tau)-u(T)}{\tau-T} \geq \frac{u(T)-u(\eta)}{T-\eta} \\
u(\tau) \leq u(T)+\frac{u(T)-u(\eta)}{T-\eta}(\tau-T) \\
u(\tau) \leq u(T)+\frac{u(T)-u(\eta)}{T-\eta}(0-T) \\
\leq u(T)\left[1-T \frac{1-\frac{2}{\alpha \eta}}{T-\eta}\right] \\
=u(T)\left[\frac{2 T-\alpha \eta^{2}}{\alpha \eta(T-\eta)}\right] .
\end{gathered}
$$

This implies that

$$
\min _{t \in[\eta, T]} u(t) \geq \frac{\alpha \eta(T-\eta)}{2 T-\alpha \eta^{2}}\|u\|
$$

This completes the proof.

\section{Main results}

In this section, we establish the existence of positive solution for the (BVP) (1.1)-(1.2).

Let

$$
E=C[0, T], \beta=\int_{0}^{T}(T-s)^{2} a(s) d s
$$

Theorem 3.1. Assume (H1) and (H2) hold and $0<\alpha<\frac{2 T}{\eta^{2}}$. If $f_{0}=0$, then the problem (1.1)-(1.2) has at least one positive solution.

Proof. From Lemma 2.1, $u$ is a solution to the boundary value problem (1.1)-(1.2) if and only if $u$ is a fixed point of operator $A$, where $A$ is defined by

$$
\begin{aligned}
A u(t)= & \frac{t}{2 T-\alpha \eta^{2}} \int_{0}^{T}(T-s)^{2} a(s) f(s, u(s)) d s \\
& -\frac{\alpha t}{3\left(2 T-\alpha \eta^{2}\right)} \int_{0}^{\eta}(\eta-s)^{3} a(s) f(s, u(s)) d s-\frac{1}{2} \int_{0}^{t}(t-s)^{2} a(s) f(s, u(s)) d s .
\end{aligned}
$$

Denote that

$$
\Omega=\left\{u \mid u \in C([0, T], \mathbb{R}), u \geq 0, \min _{t \in[\eta, T]} u(t) \geq \gamma\|u\|\right\},
$$

where $\gamma$ is defined in (2.9). Then $\Omega$ is the convex subset of $E$.

We choose $\varepsilon>0$ and $\varepsilon \leq \frac{2 T-\alpha \eta^{2}}{T \beta}$. By $f_{0}=0$, it there exists constant $M>0$, such that $f(u)<\varepsilon u$ for $0<u<M$. For $u \in \Omega$, from Lemma 2.2 and Lemma 2.4 , we have $A u(t) \geq 0$ and $\min _{t \in[\eta, T]} A u(t) \geq \gamma\|A u\|$. 
On the other hand,

$$
\begin{aligned}
A u(t) & \leq \frac{t}{2 T-\alpha \eta^{2}} \int_{0}^{T}(T-s)^{2} a(s) f(u(s)) d s \\
& \leq \frac{t}{2 T-\alpha \eta^{2}} \int_{0}^{T}(T-s)^{2} a(s) \varepsilon u(s) d s \\
& \leq\|u\| \frac{T \varepsilon}{2 T-\alpha \eta^{2}} \int_{0}^{T}(T-s)^{2} a(s) d s \\
& \leq\|u\| \leq M .
\end{aligned}
$$

Thus $\|A u\| \leq\|u\| . u \in K \cap \partial \Omega_{1}$. Hence $A \Omega \subset \Omega$. It easy to check that $A: \Omega \rightarrow \Omega$ is completely continuous. For $u \in \Omega$ and $0<\lambda<1$, we have $u(t)=\lambda A u(t)<A u(t) \leq M$, which implies $\|u\| \leq M$. So $\{u \in \Omega \mid u=\lambda A u, 0<\lambda<1\}$ is bounded. By Theorem 1.1 the operator $A$ has at least one fixed point in $\Omega$. Thus the problem (1.1)-(1.2) has at least one positive solution. The proof is complete.

Theorem 3.2. Assume (H1) and (H2) hold, and $0<\alpha<\frac{2 T}{\eta^{2}}$. If $f_{\infty}=0$, then the problem (1.1)-(1.2) has at least one positive solution.

Proof. Choose $\varepsilon<\frac{2 T-\alpha \eta^{2}}{2 T \beta}$. By $f_{\infty}=0$, we know there exists Constant $N$, such that $f(u)<\varepsilon u$ for $u>N$.

Select

$$
M \geq N+1+\frac{2 T \beta}{2 T-\alpha \eta^{2}} \max _{0 \leq u \leq N} f(u)
$$

Let

$$
\Omega=\left\{u \mid u \in C[0, T], u \geq 0,\|u\| \leq M, \min _{t \in[\eta, T]} u(t) \geq \gamma\|u\|\right\},
$$

then $\Omega$ is the convex subset of $E$. For $u \in \Omega$, by Lemma 2.2 and Lemma 2.4 we know $A u(t) \geq 0$ and $\min _{t \in[\eta, T]} A u(t() \geq \gamma\|A u\|)$. On the other hand,

$$
\begin{aligned}
A u(t) & \leq \frac{t}{2 T-\alpha \eta^{2}} \int_{0}^{T}(T-s)^{2} a(s) f(u(s)) d s \\
& \leq \frac{T}{2 T-\alpha \eta^{2}} \int_{0}^{T}(T-s)^{2} a(s) \varepsilon u(s) d s \\
& =\frac{T}{2 T-\alpha \eta^{2}} \int_{I_{1}=\{s \in[0, T], u(s)>N\}}^{T}(T-s)^{2} a(s) f(u(s)) d s+\frac{T}{2 T-\alpha \eta^{2}} \int_{I_{2}=\{s \in[0, T], u(s) \leq N\}}(T-s)^{2} a(s) f(u(s)) d s \\
& \leq \frac{T}{2 T-\alpha \eta^{2}} \int_{0}^{T}(T-s)^{2} a(s) \varepsilon u(s) d s+\frac{T}{2 T-\alpha \eta^{2}} \int_{0}^{T}(T-s)^{2} a(s) d s . \max _{0 \leq u \leq N} f(u) \\
& \leq \frac{T \varepsilon}{2 T-\alpha \eta^{2}}\|u\| \int_{0}^{T}(T-s)^{2} a(s) d s+\frac{T}{2 T-\alpha \eta^{2}} \int_{0}^{T}(T-s)^{2} a(s) d s . \max _{0 \leq u \leq N} f(u) \\
& \leq \frac{T \varepsilon}{2 T-\alpha \eta^{2}} M \int(T-s)^{2} a(s) d s+\frac{T}{2 T-\alpha \eta^{2}} \int_{0}^{T}(T-s)^{2} a(s) d s . \max _{0 \leq u \leq N} f(u) \\
& \leq \frac{T \varepsilon}{2 T-\alpha \eta^{2}} M \beta+\frac{T}{2 T-\alpha \eta^{2}} \beta \max _{0 \leq u \leq N} f(u) \\
& \leq \frac{1}{2} M+\frac{1}{2} M=M .
\end{aligned}
$$

Thus $\|A u\| \leq M$. Hence, $A \Omega \subset \Omega$. IT easy to check that $A: \Omega \rightarrow \Omega$ is completely continuous.

For $u \in \Omega$ and $u=\lambda A u, 0<\lambda<1$, we have $u(t)=\lambda A u(t)<A u(t) \leq M$, which implies $\|u\| \leq M$. So, $\{u \in \Omega: u=\lambda A u, 0<\lambda<1\}$ is bounded. By Theorem 1.1, we know the operator $A$ has at least one fixed point in $\Omega$. Thus the problem (1.1)-(1.2) has at least one positive solution. The proof is complete.

Theorem 3.3. Assume (H1) and (H2) hold, and $0<\alpha<\frac{2 T}{\eta^{2}}$. If there exists constant $\rho_{1}>0$, such that $f(u) \leq \frac{\left(2 T-\alpha \eta^{2}\right) \rho_{1}}{T \beta} T \beta$ for $0<u<\rho_{1}$, then the problem (1.1)-(1.2) has at least one positive solution.

Proof. Let $\Omega=\left\{u \mid u \in C[0,1], u \geq 0,\|u\| \leq \rho_{1}, \min _{t \in[\eta, T]} u(t) \geq \gamma\|u\|\right\}$, then $\Omega$ is the convex subset of $E$.

For $u \in \Omega$, by Lemma 2.2 and Lemma 2.4, we have

$$
A u(t) \geq 0 \text { and } \min _{t \in[\eta, T]} A u(t) \geq \gamma\|A u\| .
$$

On the other hand

$$
\begin{aligned}
A u(t) & \leq \frac{t}{2 T-\alpha \eta^{2}} \int_{0}^{T}(T-s)^{2} a(s) f(u(s)) \\
& \leq \frac{t}{2 T-\alpha \eta^{2}} \int_{0}^{T}(T-s)^{2} \frac{\left(2 T-\alpha \eta^{2}\right) \rho_{1}}{T \beta} d s=\rho_{1} .
\end{aligned}
$$

Then $\|A u\| \leq \rho_{1}$. Hence, $A \Omega \subset \Omega$. It easy to check yhat $A: \Omega \rightarrow \Omega$ is completely continuous.

For $u \in \Omega$ and $u=\lambda A u, 0<\lambda<1$, we have $u(t)=\lambda A u(t)<A u(t) \leq \rho_{1}$, which implies $\|u\| \leq d$. So $\{u \in \Omega: u=\lambda A u, 0<\lambda<1\}$ is bounded. By Theorem 1.1, we know the operator $A$ has at least one fixed point in $\Omega$. Thus the problem (1.1)-(1.2) has at least one positive solution. The proof is complete. 
Theorem 3.4. Assume (H1) and (H2) hold, and $0<\alpha<\frac{2 T}{\eta^{2}}$. If there exists constant $\rho_{2}>0$, such that $f(u) \leq \frac{\left(2 T-\alpha \eta^{2}\right) \rho_{2}}{T \beta}$ for $0<u<\rho_{1}$, then the problem (1.1)-(1.2) has at least one positive solution.

\section{Proof. Choose}

$$
d>1+\rho_{2}+\frac{T \beta}{2 T-\alpha \eta^{2}} \cdot \max _{0 \leq u \leq \rho_{2}} f(u) .
$$

Let

$$
\Omega=\left\{u \mid u \in C[0, T], u \geq 0,\|u\| \leq d, \min _{t \in[\eta, T]} u(t) \geq \gamma\|u\|\right\}
$$

then $\Omega$ is the convex subset of $E$.

For $u \in \Omega$, by Lemma 2.2 and Lemma 2.4, we know $A u(t) \geq 0$ and $\min _{t \in[\eta, T]} A u(t) \geq \gamma\|A u\|$.

On the other hand,

$$
\begin{aligned}
A u(t) & \leq \frac{t}{2 T-\alpha \eta^{2}} \int_{0}^{T}(T-s)^{2} a(s) f(u(s)) d s \\
& \leq \frac{T}{2 T-\alpha \eta^{2}} \int_{0}^{T}(T-s)^{2} a(s) f(u(s)) d s \\
& =\frac{T}{2 T-\alpha \eta^{2}} \int_{I_{1}=\left\{s \in[0, T], u(s)>\rho_{2}\right\}}(T-s)^{2} a(s) f(u(s)) d s+\frac{T}{2 T-\alpha \eta^{2}} \int_{I_{2}=\left\{s \in[0, T], u(s) \leq \rho_{2}\right\}}(T-s)^{2} a(s) f(u(s)) d s \\
& \leq \frac{T}{2 T-\alpha \eta^{2}} \int_{0}^{T}(T-s)^{2} a(s) \frac{\left(2 T-\alpha \eta^{2}\right) \rho_{2}}{T \beta} d s+\frac{T}{2 T-\alpha \eta^{2}} \int_{0}^{T}(T-s)^{2} a(s) \cdot \max _{0 \leq u \leq \rho_{2}} f(u) d s \\
& \leq \rho_{2}+\frac{T \beta}{2 T-\alpha \eta^{2}} \cdot \max _{0 \leq u \leq \rho_{2}} f(u)<d
\end{aligned}
$$

Thus $\|A u\| \leq d$. Hence $A \Omega \subset \Omega$. It easy to check that the operator $A$ is completely continuous. For $u \in \Omega$ and $u=\lambda A u, 0<\lambda<1$, we have $u(t)=\lambda A u(t)<A u(t) \leq d$, which implies $\|u\| \leq d$. So $\{u \in \Omega: u=\lambda A u, 0<\lambda<1\}$ is bounded. By Theorem 1.1, we know the operator $A$ has at least one fixed point in $\Omega$. Thus the problem (1.1)-(1.2) has at least one positive solution. The proof is complete.

\section{Examples}

Example 4.1. Consider the boundary value problem

$$
\begin{aligned}
& u^{\prime \prime \prime}(t)+\frac{t^{2} u}{t+e^{u}}=0, \quad 0<t<\frac{5}{4}, \\
& u(0)=0, u^{\prime \prime}(0)=0, u\left(\frac{5}{4}\right)=35 \int_{0}^{\frac{1}{4}} u(s) d s,
\end{aligned}
$$

where $\alpha=35, \eta=\frac{1}{4}, T=\frac{5}{4}, 0<\alpha=35<40=\frac{2 T}{\eta^{2}}, f(t, u)=\frac{u}{t+e^{u}} \in C([0, T] \times[0, \infty),[0, \infty))$ and $a(t)=t^{2}>0$ for $t \in\left[\frac{1}{4}, \frac{5}{4}\right]$. Since $f_{\infty}=0$ and from Theorem 3.2, we can get that the (4.1)- (4.2) has at least one positive solution. Consequently, we cannot apply the Krasnoselskii's fixed point theorem like in [10, 17, 19]

Example 4.2. Consider the boundary value problem

$$
\begin{aligned}
& u^{\prime \prime \prime}(t)+e^{t}\left(u-\frac{u}{\sqrt{1+u}}\right)=0, \quad 0<t<\frac{3}{4}, \\
& u(0)=0, u^{\prime \prime}(0)=0, u\left(\frac{3}{4}\right)=15 \int_{0}^{0,2} u(s) d s,
\end{aligned}
$$

where $\alpha=15, \eta=0,2=\frac{1}{5}, T=\frac{3}{4}, 0<\alpha=15<37,5=\frac{2 T}{\eta^{2}}, f(t, u)=u-\frac{u}{\sqrt{1+u}} \in C([0, T] \times[0, \infty),[0, \infty))$ and a $(t)=e^{t}>0$ for $t \in\left[\frac{1}{5}, \frac{3}{4}\right]$. Obviously $f_{0}=0$. From Theorem 3.1, the (4.3)-(4.4) has at least one positive solution. On the other hand, we have $f_{0}=1$, then the function $f$ is not superlinear. Consequently, we cannot apply the Krasnoselskii's fixed point theorem like in [10, 17, 19]

\section{Acknowledgments}

The authors want to thank the anonymous referee for the throughout reading of the manuscript and several suggestions that help us improve the presentation of the paper. 


\section{References}

[1] A. Granas, J. Dugundji, Fixed point theory, Springer-Verlag, New York, (2003).

[2] A. Guezane-Lakoud and A. Frioui, Existence of solutions of a nonlinear third order boundary value problem, Fixed Point Theory, 13(2012), 501-506.

[3] A. Lomtatidze and L. Malaguti, "On a nonlocal boundary value problem for second order nonlinear singular differential equations," Georgian Mathematical Journal, vol. 7, no. 1, pp. 133-154, 2000.

[4] A. P. Palamides, Nikolaos M. Stavrakakis, Existence and uniqueness of a positive solution for a third-order three-point boundary-value problem, Electron. J. Differential Equations 155 (2010), 1-12.

[5] A. Rezaiguia, S. Kelaiaia, Existence of a positive solution for a third-order three point boundary value problem. Matematicki Vesnik, 68(2016), 12-25.

[6] B.W. Niu, J.P. Sun and Q. Y. Ren, Two positive solutions of third-order BVP with integral boundary condition and sign-changing green's function, Volume 2015, Article ID 491423, 8 pages.

[7] F. Haddouchi, S. Benaicha; Multiple positive solutions for a nonlinear three-point integral boundary-value problem. Int. J. Open Problems Compt. Math. 8(2015), 29-42.

[8] F. Haddouchi, S. Benaicha, Positive solutions of a nonlinear three-point eigenvalue problem with integral boundary conditions, Romanian Journal of Mathematics and Computer Science. 5(2015), 202-213.

[9] G. L. Karakostas and P. Ch. Tsamatos, "Multiple positive solutions of some Fredholm integral equations arisen from nonlocal boundary-value problems," Electronic Journal of Differential Equations, vol. 2002, no. 30, pp. 1-17, 2002.

[10] H. Djourdem, S. Benaicha, Existence of positive solutions for a nonlinear three-point boundary value problem with integral boundary conditions. Acta Math. Univ. Comenianae, (2018)(to appear).

[11] J. M. Gallardo, "Second-order differential operators with integral boundary conditions and generation of analytic semigroups," Te Rocky Mountain Journal of Mathematics. 30(2000), 1265-1291.

[12] J.R.Graef, B.Yang: Positive solutions of a third order nonlocal boundary value problem. Discrete Contin. Dyn. Syst. Ser. (2008), 89-97.

[13] J. P. Sun and H. B. Li, Monotone positive solution of nonlinear third-order BVP with integral boundary conditions, Boundary Value Problems, Volume 2010, Article ID 874959, 12 pages.

[14] L. J. Gao, J. P. Sun, Positive solutions of a third-Order three-Point BVP with sign-changing green's function. Mathematical Problems in Engineering, Volume 2014, Article ID 406815, 6 pages.

[15] M.R. Grossinho, F. Minhos, Existence result for some third order separated boundary value problems, Nonlinear Anal. 47 (2001), $2407-2418$.

[16] N. Bouteraa, S. Benaicha, Triple Positive solutions of higher-order nonlinear boundary value problems. Journal of Computer Science and Computational Mathematics, Volume 7, Issue 2, June 2017, 25-31.

[17] S. Benaicha and F. Hadouchi, Positive solutions of a nonlinear fourth-order integral boundary value problem, Annals of West University of Timisoara Mathematics and Computer Science, 54, (2016), 73- 86.

[18] S. H. Li, Positive solutions of nonlinear singular third-order two-point boundary value problem. J. Math. Anal. Appl. 323(2006), 413-425.

[19] X. Liu, D. Ma, The existence of positive solution for a third-order two-point boundary value problem with integral boundary conditions, Scientific Journal of Mathematics Research. 4, February 2014, 1-7.

[20] Y. Sun, Existence of triple positive solutions for a third-order three-point boundary value problem. Journal of Computational and Applied Mathematics 221 (2008) 194-201. 